\title{
Learning from People with HIV: Their Insights are Critical to Our Response to the Intersecting COVID-19 and HIV Pandemics in Africa
}

\author{
Christine Aristide $^{1} \cdot$ Samson Okello ${ }^{2,3,4} \cdot$ Mwebesa Bwana $^{2} \cdot$ Mark J. Siedner $^{2,5,6} \cdot$ Robert N. Peck $^{1,7}$
}

Published online: 30 June 2020

(c) Springer Science+Business Media, LLC, part of Springer Nature 2020

Nearly $70 \%$ of the global population of people with HIV (PWH) live in sub-Saharan Africa, in countries that are likely to be severely impacted by the rising third wave of COVID-19. The first case of COVID-19 in Sub Saharan African was not reported until February 28, 2020 in Nigeria [1], but the number of confirmed cases is now growing exponentially (Fig. 1). To date, more than 150,000 cases and 4000 deaths from COVID-19 have been reported [2] in the region. Due to lack of testing infrastructure [3] and stigma around COVID-19 [4], the actual numbers are likely much larger. We do not know yet know if HIV infection is a biological risk factor for SARS-CoV-2 infection or severe COVID-19 disease [5]. Either way, it is readily apparent that COVID-19 and related containment measures are having a profound impact on the lives of PWH. Through conversations with PWH and HIV healthcare providers in East Africa, we have learned ways in which COVID-19 has begun to impact the lives and the care of PWH in the region. We have also seen PWH partnering with HIV healthcare providers to develop creative solutions to local challenges.

Robert N. Peck

rnp2002@med.cornell.edu

1 Center for Global Health, Weill Cornell Medical College, 402 East 67th Street, 2nd Fl., New York, NY 10021, USA

2 Department of Internal Medicine, Mbarara University of Science and Technology, Mbarara, Uganda

3 Lown Scholars Progam, Department of Global and Population Health, Harvard T.H. Chan School of Public Health, Boston, MA 02114, USA

4 Department of Medicine, University of Virginia Health Systems, Charlottesville, VA, USA

5 Medical Practice Evaluation Center, Massuchussets General Hospital, 100 Cambridge Street, Suite 1600, Boston, MA 02114, USA

6 Harvard Medical School, Boston, MA, USA

7 Departments of Medicine and Pediatrics, Weill Bugando School of Medicine, Mwanza, Tanzania

\section{HIV is a Disease of Poverty and Poverty Increases the Risk of Contracting and Dying of COVID-19}

Over-crowding, poor sanitation and indoor air pollution are known risk factors for COVID-19 spread. Poverty also contributes to COVID-19 risk by presenting impossible tradeoffs between preventing disease and sustaining access to food and medicine. Many PWH in East Africa rely on crowded public transportation for work and procurement of basic amenities. Several PWH recently lamented that, although they understand the risk, they must ride public transportation every day to work so as to pay rent and other expenses. Although aware of the benefits, many PWH cannot afford to purchase protective gear, such as masks, which help to protect against the virus. In response, we have seen many HIV clinics developing public-private partnerships with neighborhood tailors to produce masks at low prices from available scraps of material. Moreover, physical distancing is a luxury that many in East Africa do not have the option of practicing. The median household size in the region is five people with few owning homes with multiple rooms [6]. In many of these homes, hand washing poses a challenge as they have limited to no access to running water, soap or sanitizer [7]. In response, we have seen HIV clinics and local businesses produce hand sanitizers using readily available materials such as locally brewed alcohol and curl activator hair gel.

\section{The Impact of COVID-19 on Food Insecurity is Another Major Concern}

Many PWH in East Africa depend on agricultural production and unskilled manual labor for survival [6]. Shelterin-place orders reduce their ability to produce, sell and buy basic goods [8]. There have been reported cases of attempted suicide in our HIV clinics for this reason. COVID-19 could 
Fig. 1 COVID-19 cases in Africa
COVID-19 Cases on the African Content

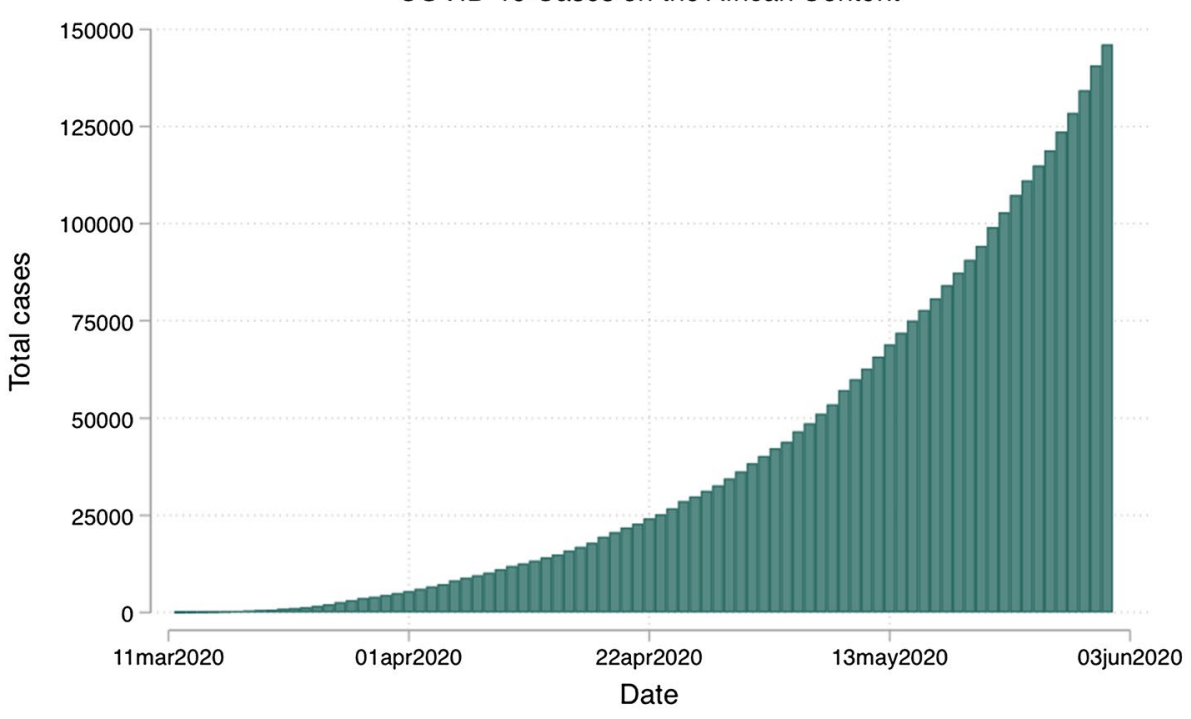

cause a stark increase in food insecurity that could kill many more people than the virus itself [9]. Concurrently, East Africa is experiencing a plague of desert locusts of a scale "unprecedented in modern times" [10] and floods that could bring an acute food shortage to over 25 million people [11]. In combination, these natural disasters are likely to affect PWH more than the general population, as PWH are more susceptible to food insecurity [12]. Food insecurity, in turn, is known to interfere with HIV care, leading to poor clinic attendance, poor antiretroviral therapy (ART) adherence, poor immunological and virologic responses, lower efficacy of ART, higher mortality and increased HIV transmission risk [13]. During the current COVID-19 crisis, for example, many PWH report being unable to take their ART on an empty stomach for fear that the drugs will make them sick.

HIV healthcare providers in Africa are partnering with PWH to develop individualized solutions to food insecurity. In some areas, national governments are providing food handouts. In other areas, local charities provide food. For some PWH, the best solution is to temporarily relocate to relatives who are in the process of harvesting their crops.

\section{COVID-19 Generates Anxiety and Fatalism, Especially Among PWH}

We are witnessing a concerning rise in feelings of helplessness among people with HIV. The proliferation of misinformation about the virus on social media feeds into these sentiments [14, 15]. Many PWH have access to smart phones and receive much of their news from WhatsApp or other mobile applications [16]. "If only I knew what to believe" complained several PWH. For example, rumors have spread that the thermal scanners used at entrances to facilities can cause brain cancer and that masks cause people to develop lifelong lung disease. There are also many messages promoting the use of herbal remedies for prevention of COVID-19 despite the fact that these herbs might adversely interact with ART [17]. The lack of understanding of coronavirus and its effects on HIV leads PWH to be more fearful, given what they know about their condition. This stress and sense of impending doom can lead to a fatalism that is reminiscent of the advent of the HIV epidemic. "If God wills it, I will die from the coronavirus," we were told by more than one PWH. This same sentiment of hopelessness debilitated PWH and families for decades at the beginning of the AIDS epidemic. HIV counselors and PWH have dealt with rumors and fatalism before, and they are using lessons learned form the past to address these new challenges. In some populations of patients, such as adolescents, counseling is more effective if conducted in groups. Some clinics are therefore planning group counseling and support sessions when the clinic is otherwise empty to allow for physical distancing and honest conversations.

\section{COVID-19 Containment Measures Can Disrupt HIV Care}

Many PWH are afraid to go to HIV clinics, fearing exposure to coronavirus. Many HIV clinics in Africa are situated within large public hospital campuses. Several PWH have informed us that they don't want to go to their HIV clinic because it is located in a hospital where coronavirus patients are admitted. In addition, many hospitals require patients to bring their own masks to gain entry and some PWH have been denied entry into hospitals because they were not able to afford a mask. For these reasons, many PWH have opted 
Fig. 2 Physical distancing in practice in an outside waiting area at an HIV clinic in East Africa

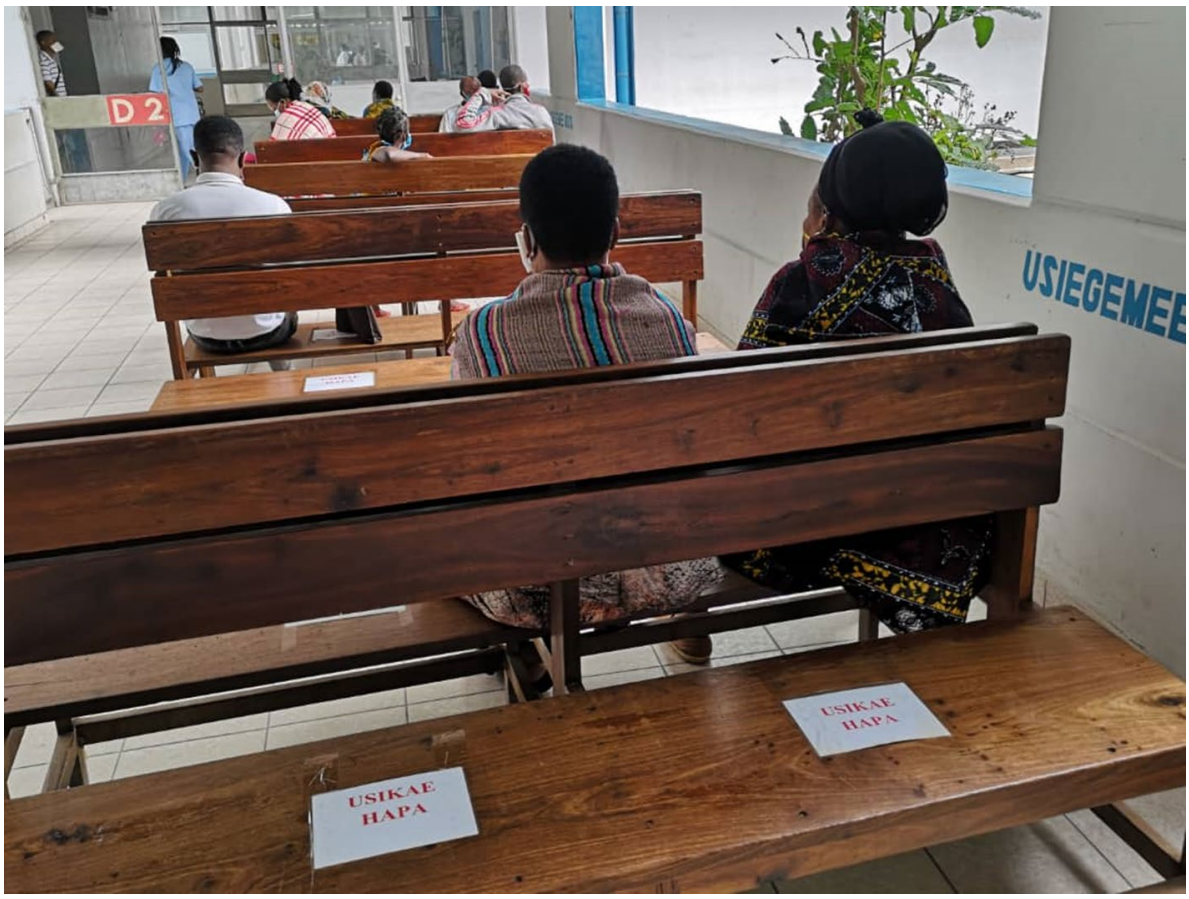

to obtain ART at health facilities closer to home. In some cases, though, this has resulted in unintended disclosure of HIV status in their communities.

In some crowded HIV clinics, strict physical distancing may not be sustainable. In response, many East African HIV clinics have moved waiting areas outside to allow for sunlight and space (Fig. 2). HIV nurses together with PWH are figuring out ways in which transportation to the HIV clinic can be made safer. Many crowded areas can be avoided, for example, with proper planning and walking or biking instead of using public transportation. When safe travel to the HIV clinic is not possible, community healthcare workers trained to adhere to COVID-19 safety protocols can delivery ART to the homes of PWH.

COVID-19 related interruptions in ART could have profound long term repercussions, setting us back after many years of progress [18]. Interrupted treatment increases the risk of treatment failure, opportunistic infections, virologic resistance, transmission of HIV from mothers to children, death and increases HIV transmission in the community [19].

Fortunately, HIV programs in sub-Saharan Africa have been at the forefront of improving efficiency of care delivery for years and they are now rising to the challenge of COVID-19 [20]. One solution that enhances efficiency of HIV care and reduces COVID-19 transmission risk is extension of ART prescription durations to 3 or more months, a strategy that was gaining traction long before the COVID-19 pandemic [21]. For countries that only allow three-month prescriptions of ART, doctors and nurses are sometimes providing two prescriptions for stable patients with suppressed viral loads so that they do not need to return for 6 months.

COVID-19 is new; but the fear, stigma, and poverty that determine health for much of the world, are not. We have much to learn from PWH about resilience and resourcefulness. As we plan the response to COVID-19 in sub-Saharan Africa, we should start by listening to PWH. Chances are they have faced similar challenges before and have solutions that are more likely to be successful in their context than global mandates designed for different settings, cultures, and resources.

Acknowledgements We dedicate this piece to all the people with HIV of East Africa from whom we have learned so much and to the healthcare workers who are bravely standing with them on the front lines of the fight against COVID-19. We would also like to thank Dr. Christopher Gordon and Mr. Karl Reis for their inspiration and assistance in putting words to paper. RP is funded by the National Institute of Health (R01 MH118107 and K01 TW010281). MJS receives funding from the National Institutes of Health (R01 AI124718, R01 AG 059504, R01 HL141053). SO receives funding from the National Institutes of Health (K43TW010715).

\section{References}

1. World Health Organization. COVID-19 Situation update for the WHO Africa region, 4 Mar 2020.

2. WHO Africa COVID-19 [Internet]. https://www.afro.who.int/ health-topics/coronavirus-covid-19. Accessed 26 May 2020.

3. Nkengasong J. Let Africa into the market for COVID-19 diagnostics. Nature. 2020;580(7805):565-565. 
4. Bruton B. Barriers to mass testing for COVID-19 in Africa. Atlantic Council; 2020.

5. Blanco JL, Ambrosioni J, Garcia F, Martínez E, Soriano A, Mallolas J, et al. COVID-19 in patients with HIV: clinical case series. Lancet HIV. 2020;7(5):e314-e316316.

6. Tanzania Ministry of Health, Tanzania National Bureau of Statistics. Demographic and Health Survey and Malaria Indicator Survey 2015-2016.

7. Riley L, Raphael E, Snyder R. A billion people live in slums. Can they survive the virus? New York Times, 2020 April

8. Food and Agriculture Organization of the United Nations. COVID-19: our hungriest, most vulnerable communities face "a crisis within a crisis." 2020.

9. Food Security Information Network (FNIS). 2020 Global Report on Food Crises.

10. Food and Agriculture Organization of the United Nations. Locusts in East Africa: a race against time; 2020.

11. Food and Agriculture Organization of the United Nations. Desert locust upsurge: progress report on the response in the Greater Horn of Africa and Yemen, January-April 2020.

12. Anema A, Vogenthaler N, Frongillo EA, Kadiyala S, Weiser SD. Food insecurity and HIV/AIDS: current knowledge, gaps, and research priorities. Curr HIV/AIDS Rep. 2009;6(4):224-31.

13. The Lancet HIV. The syndemic threat of food insecurity and HIV. Lancet HIV. 2020;7(2):e75.

14. Coronavirus: What misinformation has spread in Africa? $B B C$; 2020.
15. Supporting media to bust harmful myths on coronavirus disease. WHO Africa; 2020.

16. How WhatsApp is used and misused in Africa. The Economist; 2019.

17. Bepe N, Madanhi N, Mudzviti T, Gavi S, Maponga CC, Morse GD. The impact of herbal remedies on adverse effects and quality of life in HIV-infected individuals on antiretroviral therapy. J Infect Dev Ctries. 2011;5(1):48-53.

18. Adepoju P. Tuberculosis and HIV responses threatened by COVID-19. Lancet HIV. 2020;7(5):e319-e320320.

19. Kranzer K, Ford N. Unstructured treatment interruption of antiretroviral therapy in clinical practice: a systematic review. Trop Med Int Health. 2011;16:1297.

20. Siedner MJ, Kraemer JD, Meyer MJ, Harling G, Mngomezulu T, Gabela P, et al. Access to primary healthcare during lockdown measures for COVID-19 in rural South Africa: a longitudinal cohort study. medRxiv; 2020. https://doi. org/10.1101/2020.05.15.20103226

21. Grimsrud A, Patten G, Sharp J, Myer L, Wilkinson L, Bekker LG. Extending dispensing intervals for stable patients on ART. J Acquir Immune Defic Syndr. 2014;66(2):58-60.

Publisher's Note Springer Nature remains neutral with regard to jurisdictional claims in published maps and institutional affiliations. 